\title{
Entre « géo-patrimonologie » et muséologie géopatrimoniale: enjeux théoriques pour un futur muséal
}

Between geo-heritology and geoheritage museology: theorical issues for a museum future

\section{Fabien Van Geert}

\section{(2) OpenEdition Journals}

\section{Édition électronique}

URL : http://journals.openedition.org/iss/2234

DOI : $10.4000 /$ iss.2234

ISSN : 2306-4161

\section{Éditeur}

ICOM - International Council of Museums

\section{Édition imprimée}

Date de publication : 1 août 2020

Pagination : 193-206

ISBN : 978-2-491997-11-3

ISSN : 2309-1290

\section{Référence électronique}

Fabien Van Geert, « Entre « géo-patrimonologie » et muséologie géopatrimoniale: enjeux théoriques pour un futur muséal », ICOFOM Study Series [En ligne], 48-1 | 2020, mis en ligne le 01 août 2020, consulté le 08 août 2020. URL : http://journals.openedition.org/iss/2234 ; DOI : https://doi.org/ $10.4000 /$ iss. 2234 


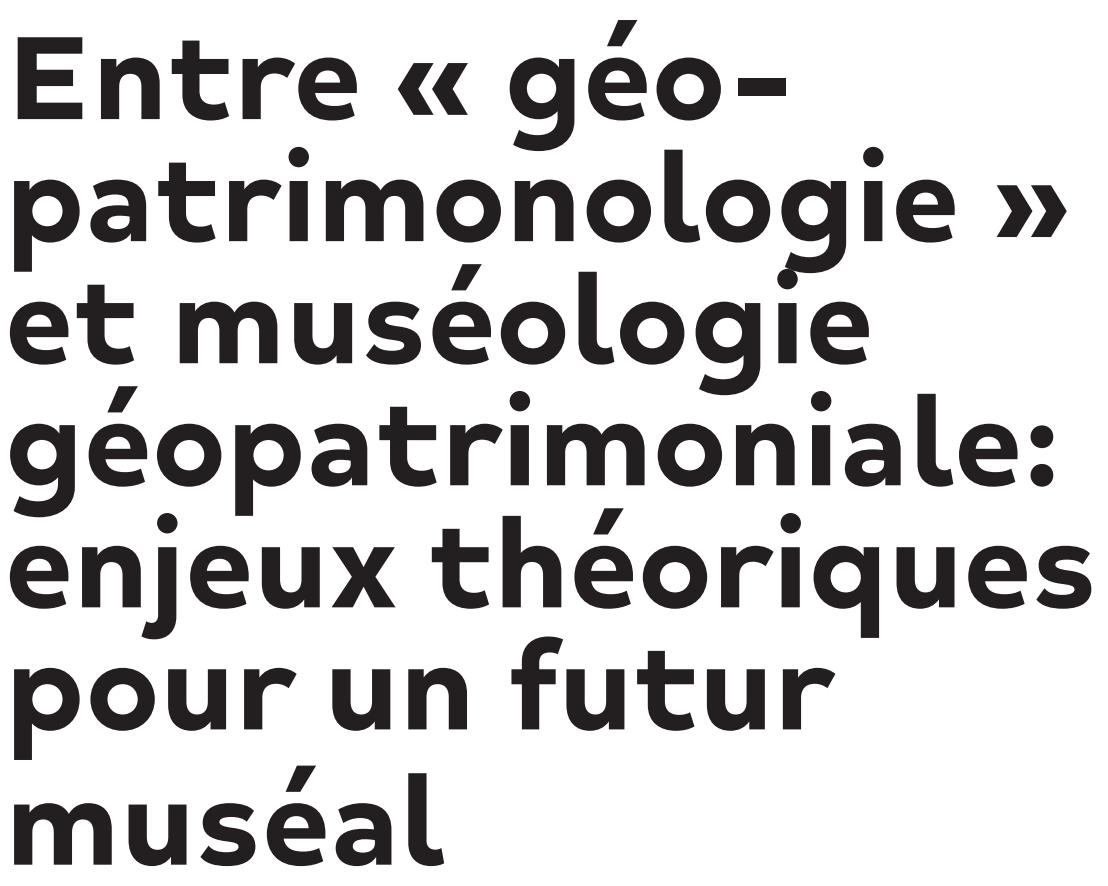

\title{
Fabien Van Geert
}

Université Sorbonne Nouvelle, Paris, France

\author{
RÉSUMÉ
}

Cet article part du postulat que l'émergence d'une nouvelle forme de patrimoine - le géopatrimoine -, a donné lieu à deux types de réflexions muséales, selon que ce dernier soit conservé in ou ex situ. Une comparaison entre ces dernières amène au constat d'une absence relative de liens entre elles, mais aussi d'un certain manque d'innovation dans l'approche muséale des institutions conservant ces collections, contrairement à l'approche in situ du géopatrimoine. L'article propose alors un renouveau de la recherche muséologique pour ces collections, en englobant les réflexions menées tant sur le géopatrimoine localisé ex situ qu'in situ afin de garantir un nouveau futur pour ces collections.

Mots clés: Géopatrimoine, musées de géologie, conservation in situ et ex situ, muséologie 


\section{Between geo-heritology and geoheritage museology: theorical issues for a museum future}

This article postulates the fact that the emergence of a new form of heritage - geo-heritage - has given rise to two types of museological reflections, depending on whether the latter is preserved in or ex situ. A comparison between these approaches leads to the observation of a relative absence of links between them, but also of a certain lack of museological innovation in the institutions conserving ex situ collections, unlike the in situ approach of geoheritage. This article thus proposes a renewal of museological researches for these collections, encompassing the reflections carried out on geoheritage preserved both ex situ and in situ in order to guarantee a new future for these collections.

Keywords: Geoheritage, geological museums, in situ and ex situ conservation, museology

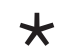

Cet article part d'un questionnement simple, mais qui touche pourtant le cour même de la réflexion théorique sur le musée : la situation ainsi que la localisation géographique des éléments considérés comme devant être conservés, - soit en tant qu'artefacts scientifiques, soit en tant qu'éléments patrimoniaux -, influencent-elles le développement de réflexions différentes selon que ces éléments se localisent in situ (sur leurs lieux d'origine) ou ex situ (conservés notamment dans les musées) ? Force est de constater en effet que, après de nombreuses années d'action professionnelle, les réflexions portant sur la conservation, les mesures de protection, mais aussi les manières de présenter ces éléments au public peuvent être différentes selon les lieux de conservation, en se consolidant d'un point de vue pratique dans des sphères professionnelles aux acteurs et aux enjeux distincts. En poussant plus loin cette logique, ces dernières auraient-elles dès lors contribué à donner lieu, d'un point de vue théorique, à deux champs de recherche distincts, entre, d'une part, les Museum Studies (pour le patrimoine conservé ex situ) et, d'autre part, les Heritage Studies (pour le patrimoine maintenu in situ)?

Cousines, sœurs ou jumelles, les relations entre ces deux approches ont souvent été débattues du fait de leurs nombreuses zones de recoupement. Davallon se propose ainsi de distinguer la muséalisation, qui institutionnaliserait l'objet comme un objet de musée, de la patrimonialisation, qui serait le processus de reconnaissance de la valeur patrimoniale de cet objet (Davallon, I995, p. I59). 
Ainsi, selon Mairesse, « si tout ce qui est muséalisé est patrimonialisé, tout ce qui est patrimonialisé n'est pas muséalisé ", alors que le réflexe patrimonial se caractériserait par sa volonté de dépasser l'unique préservation matérielle de la «vraie chose » (Mairesse, 20II, p. 254). Pour sa part, le réflexe muséal dépasserait le seul intérêt de préservation in situ de la patrimonialisation, en intégrant également l'aspect ex situ à sa réflexion. Cela donnerait dès lors lieu à des formes multiples de muséalisation, selon le contexte où est situé l'élément à mettre en valeur, bien que ces dernières constitueraient in fine des variantes d'un même processus muséal. Certains auteurs vont en effet jusqu'à dire qu'il s'agirait d'une seule et même logique, en proposant de regrouper la sphère du patrimoine et des musées au sein d'une même approche conceptuelle. C'est le cas de Šola qui suggère le développement d'une « patrimonologie », qui devrait s'intéresser à toutes les activités liées à la conservation et à la protection du patrimoine, qu’il soit muséal ou non (Šola, 20I5). Cette approche se détacherait dès lors du postulat qui ferait de la muséologie une simple composante des études patrimoniales, certains muséologues indiquant ainsi que, bien que ces processus soient semblables, ils n'en devraient pas moins être pensés différemment car liés à des enjeux très différents (Desvallées, Mairesse \& Deloche, 20II ; Chaumier, 20I6). C'est notamment le cas de la différence entre les centres d'interprétation et les musées qui, même s'ils constituent deux approches du fait muséal, présentent des caractéristiques propres, en illustrant notamment le passage d'une muséologie de l'objet à une muséologie de l'idée (Chaumier \& Jacobi, 2009).

Ainsi, tout en reconnaissant que ces deux champs de recherche se recoupent sur de nombreux aspects, il apparait notamment qu'ils se nourrissent de cadres théoriques parfois divergents. En ce qui concerne les publics, l'action patrimoniale in situ est ainsi en grande partie redevable de théories provenant du champ de l'éducation et de la pédagogie, en donnant lieu au développement d'une pratique particulière - l'interprétation - fondée sur des réflexions développées dans le champ du patrimoine naturel par des auteurs tels que Tilden ou Muir (Chaumier \& Jacobi, 2009). Même si l'on retrouve des traces de cette réflexion dans la médiation proposée par certains musées à l'air libre et écomusées (Delarge, 2000), la réflexion sur les publics dans les espaces ex situ s'est au contraire plutôt développée sur la base d'autres approches, telles que la didactique des sciences pour les musées scientifiques (Schiele, 1989), ou l'animation socioculturelle puis la médiation culturelle pour les musées d'art et d'histoire (Aboudrar \& Mairesse, 2016). La distinction entre ces deux approches apparaît notamment dans le cas d'éléments possédant à la fois des composantes conservées in situ et ex situ, où les logiques de patrimonialisation et de muséalisation peuvent suivre des logiques assez différentes. Dans la sphère naturelle, c'est le cas de la patrimonialisation ex situ du vivant, notamment des espèces animales et végétales, dont la conservation et la présentation dans des zoos, ménageries, jardins botaniques ou serres impliquent des approches différentes de celles mises en place in situ, au sein des aires protégées. C'est également le cas du patrimoine géologique qui regrouperait, selon la défi- 
nition officielle utilisée en France, «tous les objets (patrimoine ex situ) et sites (patrimoine in situ) relatifs aux disciplines des Sciences de la Terre qui présentent un intérêt exceptionnel pour la mémoire de la Terre » (Inventaire National du Patrimoine Naturel, 2019). Ainsi, alors que cette conception du patrimoine géologique intègre à la fois des composantes conservées in situ ou ex situ, les cadres théoriques ainsi que les actions menées sur ces deux aspects peuvent être très différents.

En partant de cette hypothèse, l'objectif de cet article est de comprendre ces divergences entre ces deux conceptions du patrimoine géologique à partir du cas français. Pour ce faire, le texte est divisé en trois parties. La première porte sur l'analyse de l'émergence du patrimoine géologique, en s'intéressant tout particulièrement aux actions de mise en valeur de ce dernier in situ. La deuxième partie aborde ensuite la situation des musées où sont exposées les collections géologiques, en tentant notamment de définir une série de tendances actuellement visibles au sein de ces institutions. La troisième et dernière partie de l'article vise enfin à mettre en parallèle ces deux approches in situ et ex situ, en proposant le développement d'une approche théorique muséale englobant ces deux aspects, afin de garantir un futur muséal pour les collections conservées ex situ.

\section{L'émergence d'un nouveau patrimoine}

La reconnaissance du patrimoine géologique émerge progressivement entre les années 1960 et 1980, lorsque des réflexions spécifiques se développent visant à mieux mettre en valeur les sites géologiques. Alors que le continent européen connait une nouvelle vague de patrimonialisation de la nature sur la base de critères à la fois économiques - en lien particulièrement avec le développement du tourisme -, mais aussi environnementaux - avec notamment la création de parcs nationaux -, les géologues et les géographes souhaitent en effet distinguer la protection et la mise en valeur des aspects abiotiques de la nature de ses facteurs biotiques. Ces derniers, représentant l'ensemble des interactions du vivant d'un écosystème, auraient en effet constitué la base de la définition du patrimoine naturel depuis le XIX è siècle, en les séparant de ses aspects physico-chimiques. En conséquence, en France, suite à l'application de la loi de 1976 relative à la protection de la nature, l'insistance de la communauté scientifique sur le fait que les spécificités des sites géologiques ne sont pas suffisamment prises en compte dans ces politiques de conservation mène à la création, au début des années 1980, des premières réserves naturelles à vocation géologique.

Ce n'est cependant véritablement qu’à partir des années i99o qu'est reconnu ce nouveau patrimoine, grâce tout particulièrement à l'impulsion donnée par le Symposium international sur la protection du patrimoine géologique tenu à Digne-les-Bains (France) en r99i sous le patronage du Département des Sciences de la Terre de l'UNESCO. Lors de ce dernier, plus de izo spécialistes, chercheurs et universitaires d'une trentaine de nationalités différentes se réunissent en effet pour dresser, pour la première fois, un constat mondial 
de la conservation des témoins géologiques. Ce symposium consolide dès lors l'idée du besoin de protection des sites liés aux phénomènes géologiques (tels que le volcanisme, la ségrégation magmatique, le métamorphisme, l'altération, la sédimentation, etc.), mais aussi de ceux témoignant de l'histoire de la Terre (que ce soit du point de vue de la paléontologie, de la tectonique globale, du climat, du niveau marin, etc.), jusqu'alors peu pris en compte dans les politiques patrimoniales. Avec la publication de la «Déclaration internationale des droits de la mémoire de la Terre » à l'issu de ce symposium, émerge dès lors la notion de patrimoine géologique, ou géopatrimoine. Cette notion est suivie, très rapidement, par celle de géodiversité visant à désigner, conjointement avec celle de biodiversité popularisée par la Conférence des Nations unies sur l'environnement et le développement à Rio de Janeiro en 1992, la diversité de la nature dans sa globalité. La géodiversité représenterait en effet « l'ensemble des éléments des sous-sols, sols et paysages qui, assemblés les uns aux autres, constituent des systèmes organisés, issus de processus géologiques » (Sharples, 1993), en concernant autant les phénomènes passés de la Terre observables dans les sous-sols, sols et paysages, que les phénomènes courants actuels qui agissent sur ces derniers.

A l'image d'autres éléments, la reconnaissance de ce nouveau statut passe dès lors par un processus de patrimonialisation composé de cinq étapes interdépendantes d'une « chaîne patrimoniale » constituée par la désignation, la classification, la conservation, la restauration et la publicisation (Fabre, 2013). La phase de désignation est composée par une tentative de théorisation des valeurs géopatrimoniales des sites. Reynard (2005) définit ainsi que différents types de valeurs peuvent être attribuées aux paysages géomorphologiques, telles que des valeurs économiques, scientifiques, historiques, culturelles ou encore esthétiques. La phase de classification est, quant à elle, constituée par des réflexions portant sur la réalisation des inventaires, mais aussi sur leurs modalités, ces derniers devant servir de base pour la mise en place de politiques de conservation raisonnées (De Wever et al., 20I8). À partir de cette phase, des réflexions émergent quant aux mesures de conservation et de restauration de ce géopatrimoine, toutes deux englobées désormais sous le concept de géoconservation (Pannizza, 200I), tandis que des appareils législatifs se mettent en place à différentes échelles pour mieux le protéger. Enfin, la phase de publicisation du géopatrimoine est constituée par des activités de promotion et de mise en valeur visant à « faire patrimoine » au-delà de la communauté scientifique, à partir des manières spécifiques de chaque contexte d'appréhender la géologie, soit en lien avec des valeurs spirituelles, à l'image de la Chine (Du \& Girault, 2019), soit au travers de l'interprétation de la nature, comme c'est le cas aux Etats-Unis. Parmi ces activités, notons tout particulièrement la création, dès les années 2000, de géoparcs visant à valoriser le géopatrimoine d'un point de vue scientifique, éducatif, mais aussi touristique afin de favoriser le développement économique des territoires (UNESCO, 20I9). Leur rapide reproduction ainsi que l'instauration du Programme international Géosciences et Geoparks par l'UNESCO en 2015, ouvrent notamment de nouveaux thèmes de recherche 
pour les scientifiques. Certains abordent ainsi la question du géotourisme pour désigner un nouveau type de visiteur intéressé par la mémoire de la Terre (González-Tejada, Du, Read \& Girault, 2017), tandis que d'autres tentent de comprendre le rôle économique joué par ces géoparcs (Farsani, Coelho \& Costa, 20Io), qui incarnent parfois de véritables stratégies de marketing territorial (Van Geert, 2019). Enfin, certaines références tentent d'aborder les tensions inhérentes entre les missions de ce nouveau label (Girault, 2019), tiraillé entre son impact présumé sur le développement économique et la protection effective qu'il permet du géopatrimoine.

En lien parfois avec ces géoparcs, cette phase de publicisation voit notamment la création de nombreuses activités de médiations au sein de ces territoires, telles que des balades géologiques, des journées de divulgation, des conférences, des jeux, des publications ou encore des applications numériques afin de garantir aux géotouristes une expérience de visite globale. Des modalités d'interprétation sont également proposées in situ, au travers de sentiers pédagogiques, de panneaux d'interprétation, ou encore de centres d'interprétation voire de musées. Au sein de ces actions, différentes approches originales sont observables. Outre l'approche purement géologique, c'est le cas de récits interdisciplinaires adressées au grand public, peu familier avec la géologie, cette "discipline mal aimée » (Gohau, 200I), afin de leur transmettre l'importance de protéger cette géodiversité. On y retrouve, premièrement, une volonté de proposer une interprétation didactique de la formation des paysages, au travers souvent de dessins, de vidéos ou de maquettes, afin de favoriser une compréhension de l'histoire de la Terre à partir de ses conséquences visibles sur la nature. Ces dispositifs tentent également d'aborder les dimensions socio-économiques et socio-culturelles de la géodiversité, en traitant des cultures, des manières de vivre, mais aussi des légendes locales expliquant les formes géologiques. Deuxièmement, ces dispositifs peuvent également proposer une approche esthétique de l'histoire de la Terre, au travers notamment de la réalisation d'expositions de peintures ou de photographies établissant parfois des liens formels entre les créations humaines et celles produites par la Terre. Enfin, les risques naturels de ces territoires liés à leurs spécificités géologiques peuvent également être abordés au sein de ces médiations, en tentant de la sorte d'avoir un impact social, voire politique, sur les décisions liées à l'aménagement durable du territoire de ces zones.

\section{Pendant ce temps, dans les musées...}

Face à l'affirmation de ce nouveau patrimoine, ainsi qu'à l'intérêt qu'il génère chez les élus par l'entremise des géoparcs et des actions de mise en valeur in situ du géopatrimoine, l'approche ex situ contraste en faisant moins l'objet d'intérêt. Les collections que l'on pourrait qualifier de géopatrimoniales, leur présentation et leur mise en valeur au sein des musées restent en effet très peu abordées par les chercheurs. Du côté des sciences de la Terre, où est principalement développé le concept de géopatrimoine, les plus prestigieuses revues 
de géologie ou de géographie n’abordent ainsi pas ces musées si ce n’est pour les spécimens qui y sont conservés, qui y font parfois l'objet d'études scientifiques. Ce n'est pas non plus le cas des revues portant sur le géopatrimoine. Par exemple, entre 2009 et 2019, la revue Geoheritage a publié 343 articles, mais seuls 4 de ces derniers abordent la question du géopatrimoine dans les musées, sans qu'aucun ne porte exclusivement sur la manière de l'exposer. C'est également le cas de International Journal of Geoheritage and Parks, deuxième principale revue portant sur le géopatrimoine publiée depuis 2013, où aucun article n'a depuis abordé les musées. Du côté de la muséologie, l'exposition de ces collections ne constitue pas non plus le sujet d'articles au sein des principales références de ce champ de recherche. Des revues internationales telles que ICOFOM Study Series, Curator, Museum World, Museum Management and Curatorship ou encore Culture \& Musées n'ont pas abordé ces questions, si ce n'est au travers de l'approche plus générale de la muséologie des sciences, qui ne mentionne pourtant pas explicitement la géologie. L'étude des enjeux muséologiques de ces collections semble constituer dans ce sens un angle mort de la recherche scientifique actuelle, contrairement à celle émergente portant sur le géopatrimoine in situ. Cette absence de références concernant les collections géologiques contraste pourtant avec l'importance quantitative de ces dernières au sein des réserves muséales. Les institutions européennes conservent en effet plusieurs millions d'objets illustrant les phénomènes géologiques, géomorphologiques et pédologiques ainsi que les processus naturels qui les forment et les modifient. Il s'agit notamment des muséums d'histoire naturelle, mais aussi des musées de science, des musées et centres d'interprétation de géosites, des musées pluridisciplinaires voire encore des collections privées. En France, le Muséum national d'histoire naturelle n'inventorie ainsi pas moins de 296 espaces ouverts au public conservant des collections géologiques ou préhistoriques (la distinction n'y est pas faite) (Egoroff, 20I2). Ce chiffre ne contemple en outre pas les musées universitaires dont un certain nombre ne sont pas visitables par le grand public.

Cette situation diverge dès lors de celle d'autres collections scientifiques, conservées parfois dans les mêmes institutions, qui ont fait l'objet de nombreuses études mais aussi de profondes rénovations muséales. En France, c'est tout particulièrement le cas, depuis les années 1980 , avec la rénovation des musées dépendant du Ministère de l'éducation nationale (tels que la Grande galerie de l'évolution du Muséum national d'histoire naturelle, du Musée national des techniques ou encore de la Cité des sciences et de l'industrie), parallèlement à la politique des Grands Travaux mise en place dans le même temps par le Ministère de la culture. Ces institutions tentent en effet de rompre alors avec une vision du musée de science perçu comme un espace de sacralisation du savoir, en plaçant désormais le visiteur au cœur du dispositif (Eidelman \& Van Praët, 2000). C'est notamment dans ce cadre que de nouvelles thématiques d'intérêt sociétal entrent au musée de sciences naturelles, à l'image de l'environnement (Davallon, Schiele \& Grandmont, 1992), du développement durable (Hebda, 2007; Chaumier \& Porcedda, 20II), puis de l'Anthropocène devenu l'objet de pratiques muséales particulières (Glazyrina, Zhuzhgova \& Vyguzova, 2019), 
au sein d'une véritable « révolution de la muséologie scientifique » (Schiele \& Koster, 1998) qui lui permet de se distinguer d'autres formes muséales (Schiele, 200I). Les collections ethnographiques de ces institutions sont également profondément repensées à partir des années I99o sous l'influence de la muséologie postcoloniale, largement étudiée dans le cadre de la rénovation des musées coloniaux (Van Geert, 2020).

Face à ces rénovations de fond, les collections géologiques semblent présenter, au contraire, peu de nouvelles réflexions muséologiques alors que la médiation scientifique y apparaît également moins développée, si ce n'est dans les centres de culture scientifique, technique et industrielle créés dans les années I970 en France, où les processus géologiques, géomorphologiques, mais aussi la sismologie ou la vulcanologie peuvent être présentés au public. Au-delà de ces centres, l'exposition des collections se rapproche plutôt d'une "muséologie d'idées ", basée sur une exposition du savoir scientifique à partir des objets (Davallon, I992), voire d'une «muséologie d'objets » (Davallon, I992), fondée sur une rencontre directe entre le visiteur et l'aspect notamment esthétique des objets.

Au-delà de ces approches majoritaires, souvent héritées de muséalisations mises en place dans les années 1970, on peut cependant noter dans ces musées et leurs expositions temporaires des traces de réflexions muséales basées sur une approche polysémique des collections géologiques. Bétard (2017) rappelle en effet que ces dernières ne se limitent pas à la portion abiotique du patrimoine naturel, mais qu'elles sont également chargées la plupart du temps d'une dimension culturelle, permettant de les considérer comme des « construits hybrides à l'interface entre une connaissance naturaliste ou géoscientifique, une approche sensible, une appropriation collective et/ou une décision politique » (Bétard, 20I7, p. 53I). Cette conception est également reprise par de De Wever pour qui le géopatrimoine offrirait des informations permettant la compréhension de la formation ou de l'évolution de la Terre, mais aussi de l'histoire des sciences, tout en pouvant servir des objectifs pédagogiques (De Wever et al., 2019, p.I4). En comparant entre elles ces différentes approches, il est possible de déterminer de grandes tendances dans les présentations actuelles de ces collections, que l'on retrouve parfois dans une même institution, au grès des vitrines et des salles d'expositions, selon les sensibilités des conservateurs étant intervenus. Ces approches ne sont donc en aucun cas exclusives les unes des autres, mais ont plutôt tendance à coexister au sein d'une même institution. Sans volonté d'exhaustivité, nous en décrivons ici trois.

La première, la plus commune et répandue, consiste en une approche esthétique des collections, et tout particulièrement des minéraux. Cette présentation se veut ainsi ouverte au plus large public, en ne se basant pas exclusivement sur des contenus scientifiques, mais plutôt sur une appréciation esthétique, voire artistique des formes et des couleurs des collections. Les objets emblématiques sont alors présentés comme des œuvres d'art, parfois magnifiées par une scénographie qui en fait ressortir les contours, les matières, ou encore la brillance. 
Les concepts de «trésors », «merveilles » ou encore de « chefs d'œuvre » de la Terre constituent alors souvent le titre de ces expositions. Dans certains cas, cette approche esthétique peut également se baser sur la forme cocasse de certains minéraux, qui évoquent des formes humaines ou imaginaires. Au-delà de l'aspect visuel, notons que cette mise en valeur esthétique des objets peut également constituer un moyen de médiation permettant d'expliquer au public les phénomènes géologiques, chimiques et physiques. C'est notamment le cas récurrent de la présentation de la fluorescence de certains minéraux par les musées, en projetant sur ces derniers des rayons ultraviolets.

La deuxième tendance consiste en une approche sociétale, dont le but est d'établir des liens entre les objets et les intérêts de la société contemporaine. Sous l'influence du paradigme des musées de société (Drouguet, 2015), les expositions élaborées selon cette approche tentent notamment d'établir des dialogues entre leurs collections et des objets contemporains issus parfois de la culture populaire. Notons tout particulièrement ici le cas des météorites, et plus largement de la cosmologie, dont les représentations sont nombreuses au sein des univers littéraires ou cinématographiques, comme l'explorent maintes expositions temporaires et dispositifs permanents présentant des objets de ce type dans les salles. Au-delà de cette approche, les musées peuvent également présenter leurs collections à partir du prisme des ressources naturelles, en réactualisant de la sorte la muséographie basée sur les potentielles ressources naturelles que l'on retrouvait dans certains musées universitaires aux XIX ${ }^{e}$ et $\mathrm{XX}^{\mathrm{e}}$ siècles. La présence de ces matériaux sur un territoire, leur influence sur l'aspect de ses paysages et de ses constructions, mais aussi ses modalités d'extraction et d'usage peuvent ainsi être abordées. Bien en lien avec une conception sociétale des musées perçus en tant qu'espaces citoyens, certaines institutions présentent enfin leurs collections à partir des enjeux contemporains du monde, tels de l'extraction minière et ses effets, mais aussi les risques et les catastrophes naturelles que peuvent provoquer les phénomènes géologiques, tels que des glissements de terrain ou des éruptions volcaniques. La médiation autour des enjeux de durabilité sont ainsi sous-jacents à cette optique, en tentant de favoriser, dans les murs du musée, un débat social et politique sur l'exploitation raisonnée et sur une bonne gestion de ces ressources naturelles pour préserver au mieux possible l'environnement et assurer le développement durable des sociétés.

Enfin, la troisième tendance se base sur une approche patrimoniale des collections, en les présentant comme du patrimoine scientifique. Il convient en effet d'indiquer que certains auteurs traitent du géopatrimoine ex situ dans un sens large, à l'image de Bétard (2017), pour qui ce terme recouvre l'ensemble des collections géologiques, mais aussi les archives, les publications, les représentations picturales, les cartes, carnets de terrain et autres documents manuscrits qui participent d'un intérêt patrimonial pour les géosciences. Selon cette approche, l'aspect patrimonial des collections réside tout particulièrement dans les possibilités offertes par ces dernières pour illustrer la consolidation 
des sciences comme on peut l'observer dans certains musées universitaires peu renouvelés depuis le $\mathrm{XX}^{\mathrm{e}}$ siècle. Les objets peuvent alors être présentés dans les expositions en tant qu'illustrations des progrès des géosciences, des manières dont ces dernières se sont développées et transformées, ou encore des intérêts des chercheurs à des moments donnés. Dans d'autres cas, ces collections peuvent également être présentées en tant que patrimoine local d'une zone, au travers notamment de leur exposition dans des musées identitaires, dont un très grand nombre furent créés en France au cours des années 1980, dans un contexte de première décentralisation. Dans ces espaces, la muséographie est en effet basée sur la mise en valeur de ce qui sont présentés comme les «trésors » de la collectivité, justifiant par là-même que cette dernière s'engage financièrement pour sa conservation au travers d'espaces souvent créés pour l'occasion. Dans ce cas, les objets géologiques sont alors exposés, non pas en tant que spécimens scientifiques, mais plutôt en lien avec un territoire dont ils sont issus, permettant également la mise en valeur de l'érudit ou de l'amateur local qui les a collectés et légués ensuite au musée.

Au sein de ces différentes tendances, il apparaît cependant que peu de musées présentent leurs collections sous le prisme du géopatrimoine. Ce terme reste en effet majoritairement absent des institutions, tant dans les feuilles de salles, les cartels que dans les médiations orales, et ce, même dans les expositions renouvelées récemment. Des indices peuvent néanmoins indiquer que cet aspect géopatrimonial est sous-jacent à certains des termes employés. C'est notamment le cas du concept de "trésor de la terre »utilisé par certaines institutions, qui peut renvoyer à l'idée d'un patrimoine de la Terre qu'il conviendrait de conserver au sein des espaces expositifs. L'absence explicite de ce terme reste cependant contrastante par rapport à ce qui se passe dans l'interprétation in situ des espaces géologiques vue précédemment, où son usage est particulièrement récurrent. Ainsi, même si certains points communs existent entre ces deux types de médiation, telle que l'approche interdisciplinaire du géopatrimoine in situ que l'on retrouve dans l'exposition des collections de certains musées, il convient de s'interroger sur les raisons de ce décalage mais aussi ses conséquences.

\section{Pour une approche globale du géopatrimoine}

Face à ces différences de traitement entre le géopatrimoine in situ et ex situ, il importe tout d'abord de se demander si la didactique des sciences a été suffisamment prise en compte et intégrée par les départements de sciences de la Terre des musées, où les collections semblent encore souvent pensées comme des artefacts scientifiques, ou, au mieux, comme des exemples du patrimoine scientifique. Plus profondément, cette réflexion pousse à se questionner dans quelle mesure la « révolution de la muséologie des sciences » décrite par Schiele et Koster (1998) a été intégrée dans les galeries et musées exposant la partie ex situ du géopatrimoine. On ne peut dans ce sens que déplorer le fait qu’il n'existe désormais que peu de formations en minéralogie et en géologie qui ouvrent des perspectives en muséologie, notamment en France, où les modalités 
d'exposition pourraient être pensées et questionnées. Et que dire des autres formations, où la muséologie scientifique et ses enjeux ne font l'objet que de très rares descriptions. Face à cette situation, conviendrait-il dès lors pour les galeries géologiques de se tourner vers les approches muséologiques mises en place par les autres musées de sciences naturelles? On peut noter ainsi que certaines voix s'élèvent dans ce sens, telle que celle d'Henriques (2015) qui souhaiterait voir se combiner dans les musées la géologie, la paléontologie et les sciences biologiques afin de présenter aux visiteurs une approche plus holistique de la nature. Bien que la reconnaissance du géopatrimoine se soit faite à partir de la volonté de l'autonomiser d’un patrimoine naturel plus général sur lequel étaient fondées jusqu'alors les réflexions patrimoniales et muséologiques, ce rapprochement plus intime entre les différents aspects de la muséologie scientifique permettrait-il aux collections géologiques de se réinventer, en lien notamment avec les publics?

Au-delà de cet aspect, ce décalage entre approches in situ et ex situ est sans doute également imputable au fait que les acteurs de ces deux réflexions sont souvent différents, notamment au sein du maillon de publicisation de la «chaîne patrimoniale ». En effet, là où les acteurs touristiques et les chargés de projets ont pu investir les actions de valorisation in situ, notamment dans le cadre de la mise en place de projets de territoires autour du géopatrimoine, les acteurs de la muséologie ex situ continuent souvent de travailler selon une approche disciplinaire liée en grande partie à la minéralogie, à la géologie ou à d'autres sciences connexes. Les autres chercheurs en sciences naturelles, voire ceux liées aux sciences sociales, y sont ainsi majoritairement absents, rendant sans doute difficile une ouverture dans les manières de penser l'objet muséal ainsi que les expositions.

Ces deux questionnements convergent ainsi vers le constat qu'il importe de développer la recherche théorique sur ces musées, très limitée comme nous l'avons vu, dont la richesse des collections est pourtant exceptionnelle à de nombreux égards. Ces derniers peuvent en effet rencontrer l'intérêt du public, comme en témoigne le succès de certaines expositions portant sur les météorites ou plus généralement sur la cosmologie. Ce chantier intellectuel impliquerait notamment le besoin de développer une comparaison internationale de cette situation. Au-delà du contexte français et européen, l'analyse de la situation en Amérique du Nord peut notamment s'avérer particulièrement riche pour la recherche, mais également pour son application au sein des musées. L'approche didactique y semble en effet manifeste, notamment dans les muséums d'histoire naturelle, faisant penser que la géologie est intégrée aux questionnements généraux des sciences naturelles, mais aussi de son interprétation in situ, comme on peut le voir dans les parcs nationaux où les aspects de la diversité biotique et abiotique sont pris en compte. Plus globalement, ce chantier devrait également se baser sur le développement d'une recherche muséologique englobante, semblable à la «patrimonologie » prônée jadis par Šola, qui intégrerait à la fois le géopatrimoine localisé ex situ et in situ en tant que frères jumeaux d'une 
même réflexion muséale. C'est en effet dans l'approche in situ que l'innovation muséologique semble désormais se dessiner, offrant l'opportunité à l'action ex situ de se penser. C'est sans doute à partir de ce rapprochement, et du développement d'une « géopatrimonologie », que l'on pourrait réinventer le futur des musées géologiques, à partir de leur tradition, mais aussi des avancées de notre société et ses nouveaux intérêts pour ces collections, que ces derniers soient économiques, touristiques ou sociétaux. C'est en tout cas ce à quoi nous appelons grâce à cet article. Espérons que cet appel soit entendu.

\section{Références}

Aboudrar, B.N., \& Mairesse, F. (2016). La médiation culturelle. Paris: Presses universitaires de France.

Bétard, F. (2017). Les géopatrimoines, de nouvelles ressources territoriales au service du développement local. Annales de Géographie, 717, 523-543.

Chaumier, S. (2016). Pourquoi la muséologie ne devra plus être une composante du patrimoine. Dans F. Mairesse (Ed.), Nouvelles tendances de la muséologie (pp. 67-94). Paris: La documentation Française.

Chaumier, S., \& Jacobi, D. (2009) (dirs). Exposer des idées : du musée au centre dinterprétation. Paris: Éditions Complicités.

Chaumier, S., \& Porcedda, A. (2oII). Musées et développement durable. Paris: La Documentation Française.

Davallon, J. (1992). Le musée est-il vraiment un média? Publics et Musées, 2, 99-I23.

Davallon, J., Schiele, B., \& Grandmont, G. (1992). L'environnement entre au musée. Lyon: Presse Universitaires de Lyon.

Davallon, J. (1995). Nouvelle muséologie vs muséologie ?. Dans M.R. Schärer (Ed.), Symposium Museum and Communities II, July 1995, Stavanger, Norway (pp. 153-168). http://network.icom.museum/fileadmin/user_upload/minisites/icofom/pdf/ISS\%2025\%20(1995).pdf.

De Wever, P., Egoroff, G., Cornée, A., Graviou, P., Avoine, J., \& Baillet, L. (2018). Patrimoine géologique. Inventaire national. Paris: EDP Sciences.

Delarge, A. (2000). Des écomusées, retour à la définition et évolution. Publics et Musées, I7-I8, I39-I55.

Desvallées, A., Mairesse, F., \& Deloche, B. (20II). Patrimoine. Dans A. Desvallées \& F. Mairesse (Eds), Dictionnaire encyclopédique de muséologie (pp. 42I-452). Paris: Armand Colin.

Drouguet, N. (2015). Le musée de société. De l'exposition de folklore aux enjeux contemporains. Paris : Armand Colin. 
Du, Y., \& Girault, Y. (2019). Pratiques géotouristiques et interprétation de la nature dans les géoparcs chinois : entre tensions et hybridation des cultures. Education relative à l'environnement, $15 / \mathrm{I}$, https://journals.openedition.org/ere/3393.

Egoroff, G. (2012). Les musées. Patrimoine géologique national. Page consultée le 3 novembre 2019, https://pgn.mnhn.fr/index.php?catid=I5\&blogid=I.

Eidelman, J., \& Van Praët, M. (Eds.). (2000). La Muséologie des sciences et ses publics: Regards croisés sur la grande galerie de l'évolution du Muséum d'histoire naturelle. Paris: Presses universitaires de France.

Fabre, D. (Ed.). (2013). Emotions patrimoniales. Paris: Editions de la Maison des sciences de l'homme.

Farsani, N.T., Coelho, C., \& Costa, C. (2010). Geotourism and Geoparks as Novel Strategies for Socio-Economic Development in Rural Areas. International Journal of Tourism Research, I3/I, 68-8I.

Girault, Y. (Ed.). (2019). Les géoparcs mondiaux UNESCO. Une mise en tension entre développement des territoires et mise en valeur du patrimoine. Londres: ISTE Editions.

Glazyrina, Y., Zhuzhgova, L., \& Vyguzova, E. (2019). 'Welcome to the Anthropocene!'. Where museum borders and responsibilities end? Dans K. Smeds (Ed.), The Future of Tradition in Museology. Materials for a discussion (pp. 6o-64). Paris: ICOFOM.

Gohau, G. (200I). La géologie, discipline mal aimée. Dans N. Hulin (Ed.), Etudes sur l'enseignement des sciences physiques et naturelles. Cahiers d'histoire et de philosophie des sciences, 49 (pp. 67-79). Lyon: ENS éditions.

González-Tejada, C., Du, Y., Read, M., \& Girault, Y. (2017). From nature conservation to geotourism development: Examining ambivalent attitudes towards UNESCO directives with the global geopark network. International Journal of Geoheritage, 5/2, I-20.

Hebda, R. J. (2007). Museums, Climate Change and Sustainability. Museum Management and Curatorship, 22/4, 329-336.

Henriques, M.E. (2015). Framing the Palaeontological Heritage Within the Geological Heritage: An Integrative Vision. Geoheritage, 7/3, 249-259.

Inventaire National du Patrimoine Naturel (2019). La géodiversité-patrimoine géologique. Inventaire National du Patrimoine Naturel. Page consultée le 8 novembre 20I9, https://inpn.mnhn.fr/informations/geodiversite/patrimoine-geologique.

Mairesse, F. (20II). Muséalisation. Dans A. Desvallées et F. Mairesse (Eds.), Dictionnaire encyclopédique de muséologie (pp. 251-269). Paris: Armand Colin. 
Pannizza, M. (200I). Geomorphosites: concepts, methods and example of geomorphological survey. Chinese Science Bulletin, 46/r, 4-5.

Reynard, E. (2005). Géomorphosites et paysages. Géomorphologie, II/3, I8I-I88.

Schiele, B. (1989) (dir.). Faire voir, faire savoir: la muséologie scientifique au présent. Québec: Musée de la civilisation.

Schiele, B. (200I). Le musée de sciences. Montée du modèle communicationnel et recomposition du champ muséal. Paris: L'Harmattan.

Schiele, B., \& Koster, E.H. (1998). La révolution de la muséologie des sciences: vers les musées du XXIe siécle? Lyon: Presses universitaires de Lyon.

Sharples, C. (1993). A methodology for the identification of significant landforms and geological sites for geoconservation purposes. Hobart: Forestry Commission.

Šola, T. (2015). Mnemosophy. An Essay on the Science of Public Memory. Zagreb: European Heritage Association.

UNESCO (2019). Qu'est ce quiun parc mondial UNESCO? UNESCO, Sciences de la Terre. Page consultée le 8 novembre 2019, http://www.unesco.org/new/ $\mathrm{fr} /$ natural-sciences/environment/earth-sciences/unesco-global-geoparks/ frequently-asked-questions/what-is-a-unesco-global-geopark/.

Van Geert, F. (2020). Du musée ethnographique au musée multiculturel. Chronique d'une transformation globale. Paris: La Documentation Française.

Van Geert, F. (2019). The uses and challenges of the geopark label as a place branding tool. The case of the Geopark of the Tremp Basin-Montsec (Catalonia, Spain). International Journal of Geoheritage and Parks, 7/2, 72-84. 